\title{
Cep57, a NEDD1-binding pericentriolar material component, is essential for spindle pole integrity
}

\author{
Qixi $\mathrm{Wu}^{1, *}$, Runsheng $\mathrm{He}^{1,{ }^{*}}$, Haining Zhou ${ }^{1}$, Albert $\mathrm{CH} \mathrm{Yu}{ }^{2}$, Bo Zhang ${ }^{1}$, Junlin Teng ${ }^{1}$, Jianguo Chen ${ }^{1,3}$ \\ ${ }^{I}$ The State Key Laboratory of Biomembrane and Membrane Bioengineering and The Key Laboratory of Cell Proliferation and Dif- \\ ferentiation of Ministry of Education, College of Life Sciences, Peking University, Beijing 100871, China; ${ }^{2}$ Department of Neurobi- \\ ology, Neuroscience Research Institute, School of Basic Medical Sciences, Peking University, Beijing 100191, China; ${ }^{3}$ The Center \\ for Theoretical Biology, Peking University, Beijing 100871, China
}

Formation of a bipolar spindle is indispensable for faithful chromosome segregation and cell division. Spindle integrity is largely dependent on the centrosome and the microtubule network. Centrosome protein Cep57 can bundle microtubules in mammalian cells. Its related protein (Cep57R) in Xenopus was characterized as a stabilization factor for microtubule-kinetochore attachment. Here we show that Cep57 is a pericentriolar material (PCM) component. Its interaction with NEDD1 is necessary for the centrosome localization of Cep57. Depletion of Cep57 leads to unaligned chromosomes and a multipolar spindle, which is induced by PCM fragmentation. In the absence of Cep57, centrosome microtubule array assembly activity is weakened, and the spindle length and microtubule density decrease. As a spindle microtubule-binding protein, Cep57 is also responsible for the proper organization of the spindle microtubule and localization of spindle pole focusing proteins. Collectively, these results suggest that Cep57, as a NEDD1binding centrosome component, could function as a spindle pole- and microtubule-stabilizing factor for establishing robust spindle architecture.

Keywords: Cep57; centrosome; spindle; cell cycle

Cell Research (2012) 22:1390-1401. doi:10.1038/cr.2012.61; published online 17 April 2012

\section{Introduction}

The centrosome, comprising two centrioles and a surrounding pericentriolar material (PCM), is the primary microtubule-organizing center (MTOC) in animal cells. After duplication of the centrosome in interphase, subsequent mitosis centrosome maturation and separation is required for orchestrating the bipolar spindle microtubule arrays [1]. At the onset of mitosis, the centrosomes generate a large number of microtubules, which form the mechanical structure of the spindle $[2,3]$. Thus, the centrosome must form a strong structure that can withstand

*These two authors contributed equally to this work.

Correspondence: Junlin Teng ${ }^{\mathrm{a}}$, Jianguo Chen ${ }^{\mathrm{b}}$

${ }^{a}$ Tel: +86-10-62767044; Fax: +86-10-62755786

E-mail: junlinteng@pku.edu.cn

${ }^{\mathrm{b}}$ Tel: +86-10-62755786; Fax: +86-10-62755786

E-mail: chenjg@pku.edu.cn

Received 28 December 2011; revised 1 February 2012; accepted 6 March

2012; published online 17 April 2012 the spindle microtubule-based tensions during mitosis. The loss of centriole cohesion and PCM factors, such as astrin [4], Kizuna [5] and tastin [6], leads to centriole disengagement and PCM fragmentation. The collapse of the centrosome yields new MTOCs and multiple spindle poles. Thus, abnormalities in number, structure and composition of the centrosome frequently result in abnormal spindle formation.

The spindle microtubule architecture is also governed by microtubule-associated proteins (MAPs), including motor [7] and non-motor MAPs [8] at the spindle poles and kinetochores and along spindle microtubules, which modulate spindle microtubule organization spatially and temporally to determine spindle morphology. The spindle pole proteins NuMA [9] and TPX2 [10] focus the spindle microtubule minus end, while microtubule-dependent motor proteins Eg5 [11], MCAK [12] and the dynein complex [9] generate motor force in the microtubule and transport mitotic proteins to ensure spindle assembly. Defects in spindle MAPs often disrupt the balance of spindle microtubule-mediated forces, which in turn affect 
the overall spindle assembly.

Cep57, originally named translokin, was shown to be involved in cytoplasmic FGF-2 intracellular trafficking $[13,14]$ and was identified as a centrosome component by a proteomics screen [15]. There are two Cep57 family members in vertebrate cells, Cep57 and Cep57-related protein (Cep57R) [16]. Xenopus Cep57 (xCep57), a Cep57R homologous protein, is localized to the kinetochore and centrosome and required for stable microtubule-kinetochore attachment and centrosomemicrotubule anchorage [17]. Mammalian Cep57 is a multidomain centrosome protein with an unconventional $\mathrm{N}$-terminal centrosome-localization domain and a Cterminal microtubule-binding domain, which is not localized to the kinetochore. Exogenously expressed Cep57 can bind, bundle and stabilize microtubules [16]. Thus, the two Cep57 family members may have different functions during mitosis. Genetic analysis showed that Cep57 mutations can cause mosaic variegated aneuploidy (MVA) syndrome [18]. The precise localization of Cep57 at the centrosome and its role during mitosis in mammalian cells remain elusive. Here, we show that Cep57 acts as a PCM component through binding to NEDD1 and is required for spindle microtubule organization and maintenance of spindle pole integrity.

\section{Results}

Cep57 is a PCM component and is associated with NEDD1

We generated polyclonal mouse and rabbit antibodies against recombinant mouse Cep57. Immunoblotting of HeLa cell lysates with the affinity-purified antibody recognized a specific band with the expected molecular weight (Supplementary information, Figure S1A). Immunostaining of HeLa cells showed that Cep57 localized within the PCM proteins pericentrin and $\gamma$-tubulin (Figure $1 \mathrm{~A}$ and Supplementary information, Figure S1B), and localized adjacent to centrin-2, a marker of the centrioles (Figure 1B). The centrosome localization of Cep57 was irrespective of cell-cycle status (Supplementary information, Figure S1B and S1C) and intact microtubule network (Supplementary information, Figure S1D). Immunoelectron microscopy showed that Cep57 was localized to the electron-dense area around centrioles (Figure 1C, arrowheads and Supplementary information, Figure S1E and S1F), but not on the centriolar microtubule wall. The above data suggested that Cep57 is a PCM component rather than a centriole protein.

To identify the centrosome-binding partner of Cep57, we performed immunoprecipitation (IP) experiments with the antibody against Cep57. The $\gamma$-TuRC components $\gamma$-tubulin, GCP2 and NEDD1 were precipitated by the Cep57 antibody, whereas other representative centrosome components, pericentrin, centrin and ninein were not precipitated (Figure 1D). Among the tested $\gamma$-TuRC components, NEDD1 appeared to have the strongest binding affinity to Cep57. Immunostaining of HeLa cells showed that Cep57 colocalized extensively with NEDD1 at the centrosome (Figure 2A). To further investigate their interaction at the centrosome, we performed fluorescence resonance energy transfer (FRET) analyses in HeLa cells probed by Cep57 (Alexa Fluor 488) and NEDD1 (Alexa Fluor 568) using an acceptor photobleaching assay. Compared with the control cells probed by Cep57 and centrin-2, the fluorescence intensity increased after NEDD1 bleaching (Figure 2A and 2B), suggesting that the NEDD1-Cep57 FRET signal was not the consequence of experimental error. The presence of FRET confirmed the specific interaction between Cep57 and NEDD1 (Figure 2B).

We then sought to find the region of NEDD1 that enabled the interaction with Cep57. NEDD1 consists of

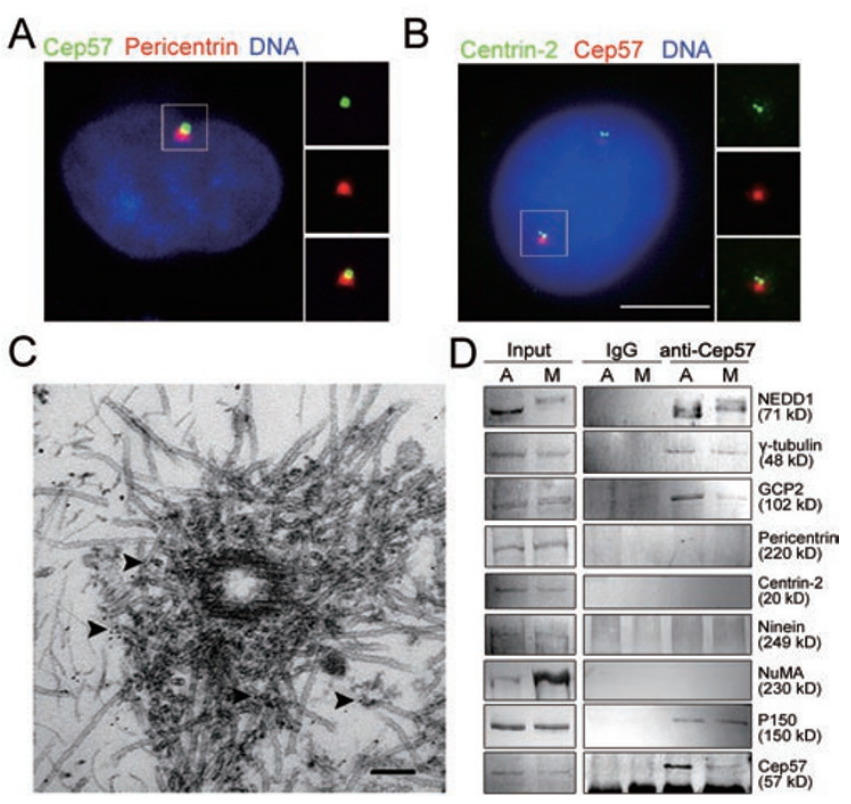

Figure 1 Cep57 is a PCM component. (A, B) Immunostaining of Cep57 (green, A; red, B), pericentrin (red, A) or centrin-2 (green, B) in HeLa cells. Nuclear DNA was stained with DAPI (blue) in all figures of this paper. Boxed regions, close-up of the centrosome. Bar, $5 \mu \mathrm{m}$. (C) Immunoelectron microscopy images of HeLa cell centrosome. Arrowheads indicate $5 \mathrm{~nm}$ gold particles. Bar, $100 \mathrm{~nm}$. (D) Cep57 is associated with $\gamma$-tubulin ring complex components. Asynchronized (A) or metaphase-arrested (M) HeLa cell lysates were immunoprecipitated with anti-Cep57 antibody or non-specific rabbit IgG. The inputs (left) and immunoprecipitants (right) were immunoblotted with the indicated antibodies. 

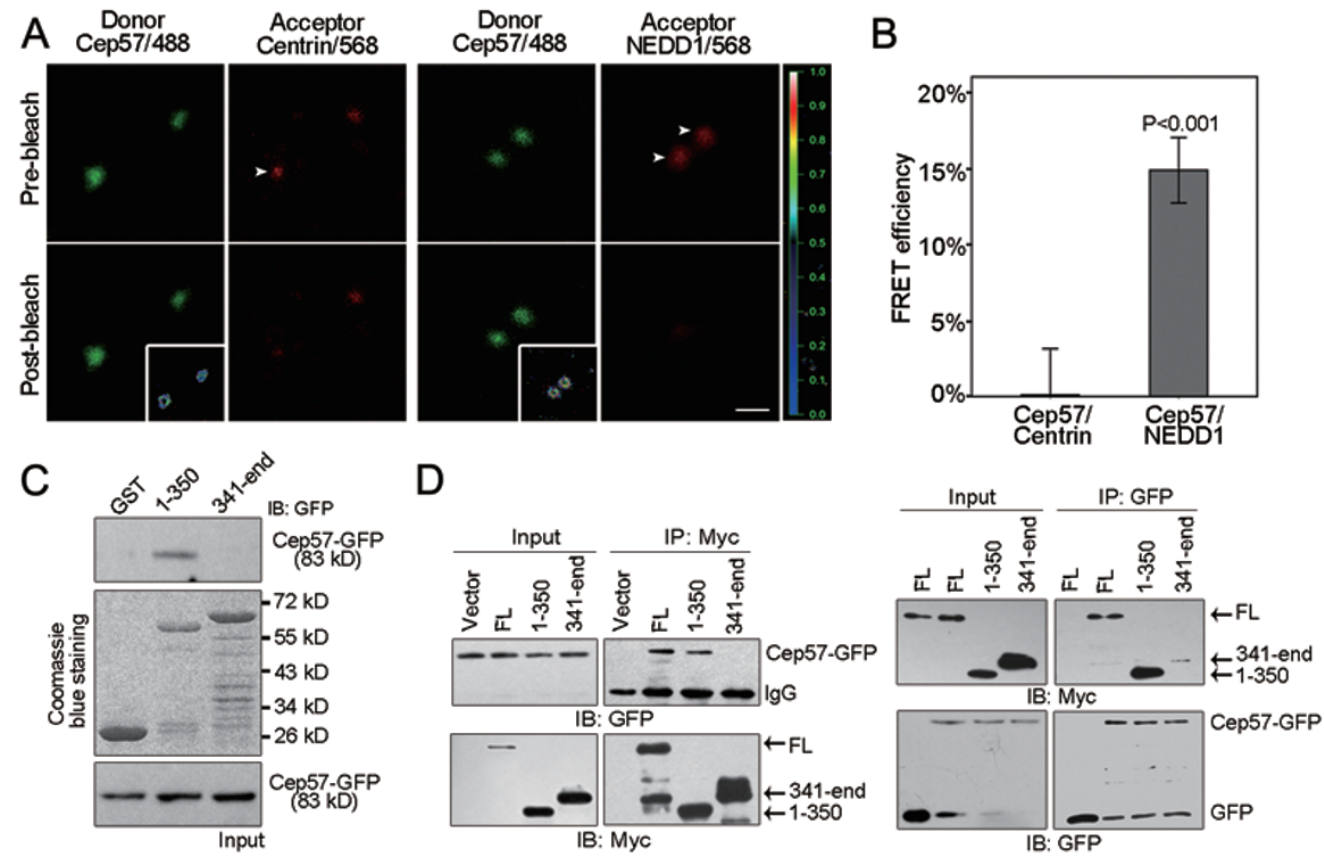

Figure 2 Cep57 binds to the N-terminus of NEDD1. (A, B) Immunostaining of Cep57 (Alexa Fluro 488) and centrin-2 or NEDD1 (Alexa Fluro 568) in HeLa cells, which were subjected to acceptor photobleaching. Representative images show the pre- and post-photobleaching states of the centrosome. Insets show the FRET intensities encoded by using the scale bar on the right. Bar, $1 \mu \mathrm{m}$. The graph shows the FRET efficiencies. $n=10$. Error bars represent mean \pm SEM. (C) Lysates of $293 T$ cells expressing Cep57-GFP were incubated with glutathione-agarose beads coated with GST or GST-NEDD1 fusion protein. The inputs and the proteins bound to the beads were immunoblotted with anti-GFP antibody. (D) Co-IP with anti-Myc and antiGFP antibodies were performed in lysates of 293T cells coexpressing Cep57-GFP and Myc vector or Myc-NEDD1 constructs, followed by immunoblotting with the indicated antibodies. FL, Myc-NEDD1 full-length. 1-350, Myc-NEDD1 (1-350). 341-end, Myc-NEDD1 (341-end).

an N-terminal multiple WD-repeat domain (NTD) and a C-terminal coiled-coil domain (CTD) [19, 20]. We performed a GST pull-down assay to test the interaction between exogenously overexpressed Cep57-GFP and GSTNEDD1-NTD (1-350) or GST-NEDD1-CTD (341-end). The result revealed the interaction between NEDD1NTD and Cep57 (Figure 2C). Coimmunoprecipitations (Co-IP) further confirmed the interaction (Figure 2D).

The Cep57-NEDD1 interaction is necessary for the centrosome localization of Cep57

To further investigate the interdependence between Cep57 and NEDD1 at the centrosome, we examined the localization pattern of Cep57-GFP in NEDD1-overexpressing cells. For quantification, we classified the exogenous Cep57-GFP localization pattern into three types: (1) extensive filaments that formed a basket-like microtubule structure around the nucleus; (2) scattered filaments along microtubules; and (3) centrosome localization (Figure 3A). Compared with control cells, the proportion of the centrosome-localized phenotype increased by more than $30 \%$ in Cep57 and NEDD1 coexpressing cells (Figure 3B). This indicated that NEDD1 may recruit Cep57 to the centrosome.

We then transfected HeLa cells with small interfering RNA (siRNA) duplexes targeted to Cep57 and NEDD1 transcripts. Their expression levels were significantly reduced and became undetectable in more than $90 \%$ of siRNA-treated cells by immunofluorescence microscopy. Immunoblotting results showed that the reduction of Cep57 protein levels did not alter the level of NEDD1 (Figure 3C), while the level of Cep57 was decreased after NEDD1 knockdown (Figure 3D). Addition of MG132, a proteasome inhibitor, rescued the reduction of Cep57 in NEDD1-depleted cells, which indicated that Cep57 would be degraded without the protection of NEDD1 (Figure 3D). Consistent with the western blot results, the depletion of Cep57 had no effect on the centrosome localization of NEDD1 (Figure 3E-3G), whereas the fluorescence intensity of Cep57 at the centrosome significantly decreased after NEDD1 depletion (Figure $3 \mathrm{H}$ and $3 \mathrm{I})$. 
A

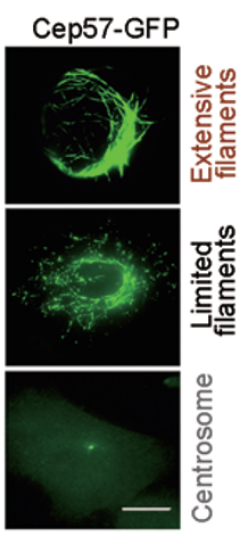

E

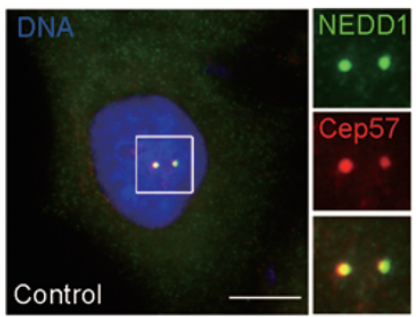

$\mathrm{H}$

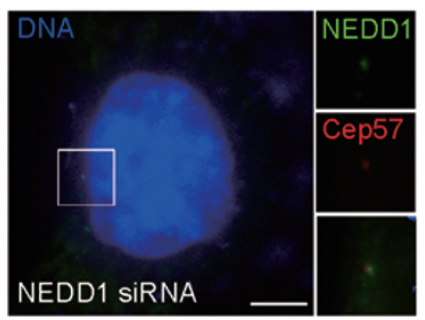

B

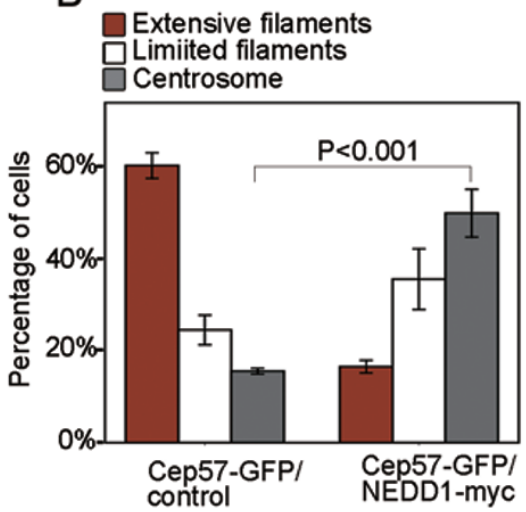

C

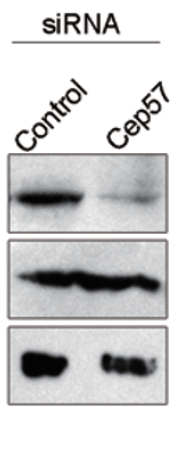

D

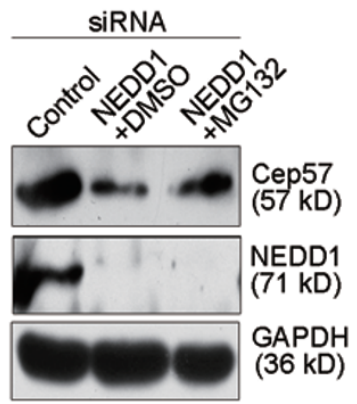

G

$$
\text { I }
$$

$\mathrm{F}$
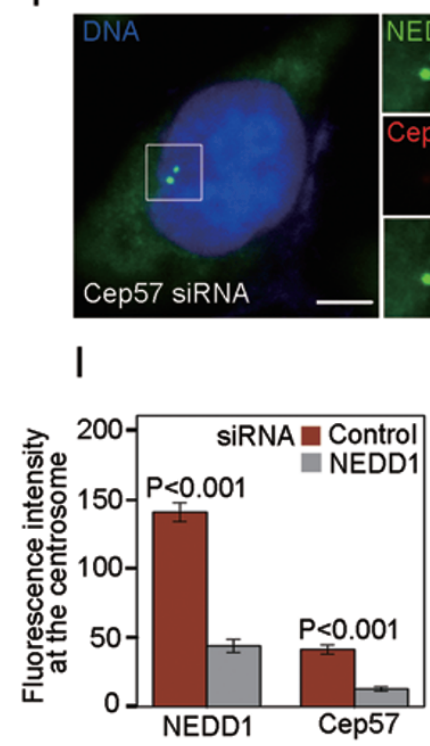

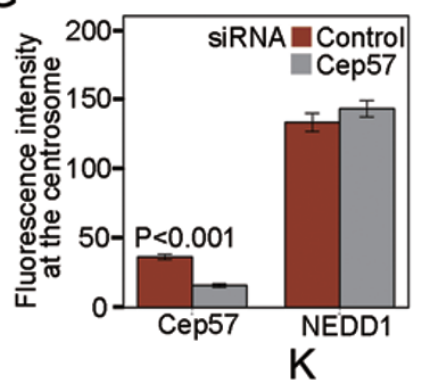

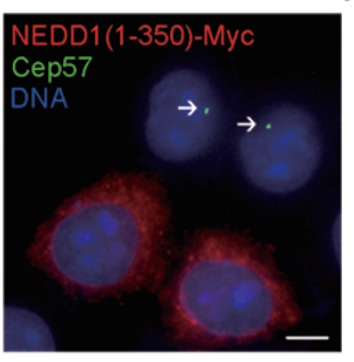

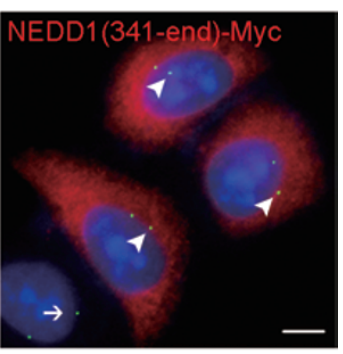

Figure 3 The centrosome localization of Cep57 requires its interaction with NEDD1. (A) Representative phenotypes of Cep57-GFP-transfected cells. Bar, $10 \mu \mathrm{m}$. (B) The graph shows the proportion of cells with indicated phenotypes $(n=3$; $>$ 80 cells per experiment). Error bars represent mean \pm SEM. (C) At $72 \mathrm{~h}$ after Cep57 siRNA transfection, the protein levels of Cep57 and NEDD1 in HeLa cells were examined by immunoblotting with the indicated antibodies. (D) At $65 \mathrm{~h}$ after NEDD1 siRNA transfection, HeLa cells were subjected to DMSO or MG132 treatment. Protein expression of Cep57 and NEDD1 was examined by immunoblotting with the indicated antibodies. (E-I) NEDD1 RNAi results in the loss of Cep57 from the centrosome. (E, F, H) Immunofluorescence images of HeLa cells treated with the indicated siRNAs. NEDD1 (green), Cep57 (red). $(G, I)$ Quantification of the fluorescence intensities within the centrosome ( $n=3 ;>60$ cells per experiment). Error bars represent mean \pm SEM. (J, K) Immunostaining of Cep57 (green) in HeLa cells transfected with NEDD1 truncates (red). Arrows and arrowheads indicate the centrosomes in untransfected and transfected cells, respectively. Bars, $5 \mu \mathrm{m}$.

Next, we tested whether the NEDD1 truncates could interfere with the centrosome localization of Cep57 and whether NEDD1 is related to the centrosome localization of Cep57. Western blot results showed that overexpressed NEDD1 full-length (NEDD1-FL) and NEDDNTD, which can bind Cep57, increased the level of Cep57, whereas NEDD1-CTD did not (Supplementary information, Figure S2). Overexpressed NEDD1-NTD and -CTD had diffuse localizations within the cytoplasm, which is consistent with previous observations [21]. Expression of the NEDD1-NTD truncate, as a dominantnegative mutant, led to a dramatic decrease of Cep57 levels at the centrosome (Figure 3J). In contrast, expression of NEDD1-CTD, which was unable to bind Cep57, did not affect the centrosome localization of Cep57 (Figure $3 \mathrm{~K})$. Taken together, the Cep57-NEDD1 interaction may protect Cep57 from degradation and be indispensable for the centrosome localization of Cep57. 
Depletion of Cep57 causes spindle pole fragmentation

We found that more than $50 \%$ of mitotic metaphase cells displayed visible spindle assembly defects, including multipolar spindles and unaligned chromosomes, $48 \mathrm{~h}$ after Cep57 siRNA treatment (Figure 4A-4C). Consistent with these defects, the average mitosis time span was significantly increased from $95 \mathrm{~min}$ in control cells (Supplementary information, Movie S1) to $215 \mathrm{~min}$ in Cep57-depleted cells (Supplementary information, Movies S2-S4). The overexpression of NEDD1-NTD, which interferes with the centrosome localization of endogenous Cep57, also induced an increased amount of cells with spindle defects (Figure 4D). Thus, Cep57 and its proper centrosome localization are critical to proper spindle establishment.

Formation of a multipolar spindle is normally caused by abnormal amplification of the centrosome, cytokinesis failure, de novo assembly of extra spindle poles or spindle pole fragmentation [22]. We examined whether Cep57 depletion would lead to centrosome over-duplication. Centriole numbers were assessed at $60 \mathrm{~h}$ posttransfection by centrin-2 staining. The Cep57 depletion increased the percentage of cells with unduplicated centrioles $(\leq 2$ per cell) compared with the controls (Supplementary information, Figure S3A). Moreover, depletion of Cep57 markedly suppressed the centriole over-amplification induced by hydroxyurea (HU) (Supplementary information, Figure S3B and S3C), which could uncouple the centrosome duplication cycle from the cell cycle [23]. Furthermore, the majority of Cep57-depleted cells did not show extra centrosomes during interphase (Supplementary information, Figure S3A), indicating that multipolar spindle formation was not a consequence of centriole over-duplication and cytokinesis failure. In addition, live-cell microscopy showed that a small ectopic pole budded from one centrosome pole (Figure 4E,
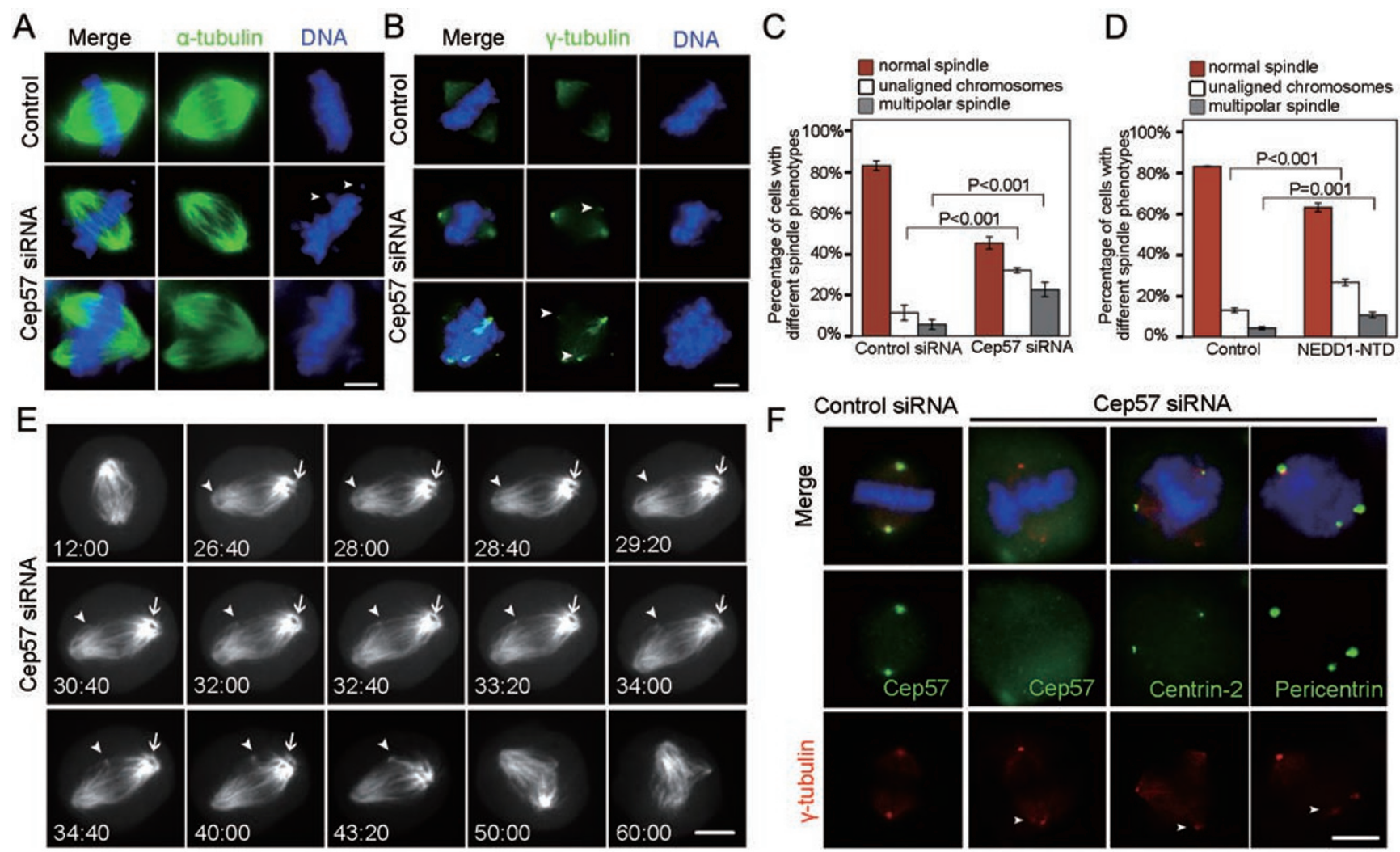

$\mathrm{F}$
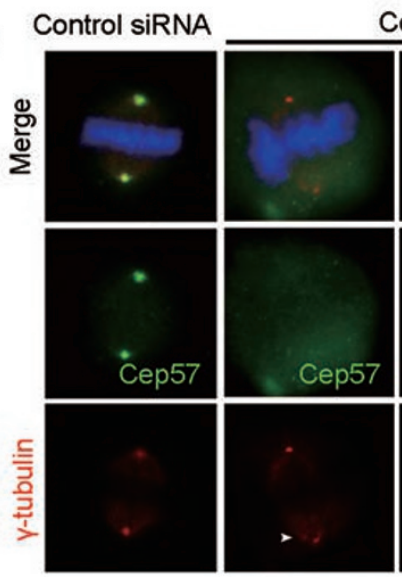

Cep57 siRNA

Figure 4 Depletion of Cep57 causes spindle assembly defects and PCM fragmentation. (A, B) Immunostaining of $\alpha$-tubulin (green, A) or $\gamma$-tubulin (green, B) in HeLa cells. Arrowheads indicate unaligned chromosomes (A) or extra spindle poles (B). (C, D) Quantification of different spindle phenotypes in synchronized metaphase HeLa cells after siRNA treatment (C) or NEDD1NTD transfection (D) ( $n=3$; > 100 cells per experiment). Error bars represent mean \pm SEM. (E) Representative time-lapse images of the mitotic progression of HeLa cells cotransfected with Cep57 siRNAs and $\alpha$-tubulin-pCAsalGFP of Supplementary information, Movie S2. Arrowheads indicate a budded pole. Arrows indicate an unfocused spindle pole. (F) Immunostaining of metaphase siRNA-transfected HeLa cells for Cep57, centrin-2 or pericentrin (green) and $\gamma$-tubulin (red). Arrowheads indicate extra spindle poles. 
arrowheads and Supplementary information, Movie S2), suggesting that the extra spindle pole did not derive from de novo assembly.

Spindle pole fragmentation can be caused by either an aberrant centriole split or PCM fragmentation. Consistent with the time-lapse result, the extra spindle poles in Cep57-depleted cells usually contained smaller $\gamma$-tubulin staining areas associated with less microtubules (Figure $4 \mathrm{~A}$ and $4 \mathrm{~B}$; arrowheads in B and Supplementary information, Movies S2-S4). In Cep57 siRNA-treated cells, pairs of centrioles were localized to only two spindle poles and centrin- 2 staining on extra $\gamma$-tubulin foci was negative (Figure 4F, arrowheads). In contrast, the PCM protein pericentrin was localized to all $\gamma$-tubulin-positive foci. Thus, the formation of a multipolar spindle caused by the depletion of Cep57 was a consequence of PCM fragmentation at the spindle poles.

Spindle forces are responsible for the spindle pole fragmentation in Cep57-depleted cells

Optimal spindle organization requires spindle microtubule forces. Eg5, a kinesin involved in the segregation of microtubule asters during prometaphase, is required for bipolar spindle formation [11]. We used monastrol
(MA) [24], a cell-permeable inhibitor of Eg5 [25], to determine whether the multipolar spindle formation induced by Cep57 depletion required Eg5. We observed that MA-treated Cep57-depleted cells showed no difference from the controls, with two centrosomes localized to the center of monopolar spindles (Figure 5A and 5B). Since the effect of MA is reversible, the MA-treated cells were replenished with fresh culture medium. After $1 \mathrm{~h}$ of MA removal, 24\% of Cep57-depleted metaphase cells restored multipolar spindles (Figure 5B). Furthermore, we eliminated K-fiber formation by depleting hNuf2 to attenuate kinetochore microtubule-mediated forces [26] (Figure 5C). The percentage of cells with multipolar spindles caused by Cep57 depletion also decreased with hNuf2 siRNA transfection (Figure 5D and 5E). Therefore, spindle forces are required for centrosome abnormalities induced by Cep57 depletion.

\section{Cep57 contributes to spindle microtubule organization}

Proper spindle microtubule organization is associated with maintenance of spindle pole integrity. To determine the role of Cep57 in microtubule organization, we examined the microtubule network assembly following Cep57 depletion. Endogenous Cep57 was successfully knocked
A

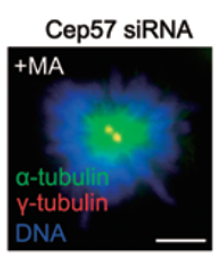

C

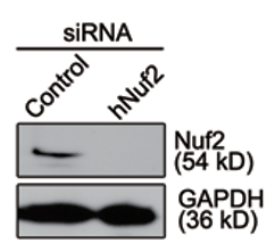

B

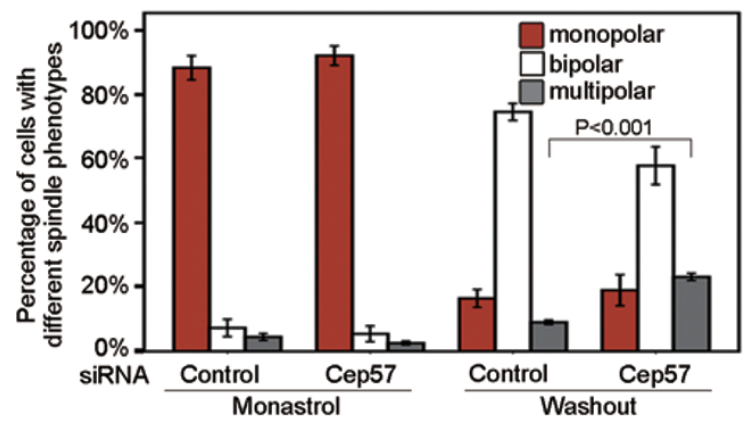

E

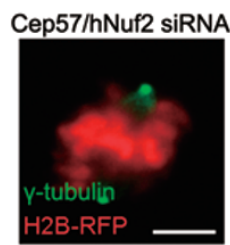

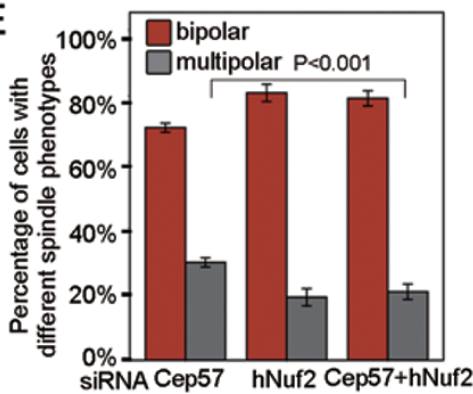

Figure 5 Spindle forces are responsible for the formation of multipolar spindles induced by Cep57 depletion. (A, B) Immunofluorescence images of MA-treated Cep57-depleted cells with $\alpha$-tubulin (green) and $\gamma$-tubulin (red) staining (A). The proportion of mitotic cells with different phenotypes was quantified after MA treatment or MA washout $(n=3$; > 200 cells per experiment). Error bars represent mean \pm SEM. (C) At $72 \mathrm{~h}$ after siRNA transfection, levels of hNuf2 in HeLa cells were examined by immunoblotting. (D, E) Representative images of Cep57 and hNuf2 siRNA-transfected cells with $\gamma$-tubulin (green) staining (D). H2B-RFP was used as a transfection marker. Bars, $5 \mu \mathrm{m}$. The proportion of mitotic cells with different phenotypes was quantified $(n=3 ;>100$ cells per experiment). Error bars represent mean \pm SEM. 
down in CHO cells by RNA interference (RNAi; Supplementary information, Figure S4A and S4B). A nocodazole recovery assay showed that microtubule fluorescence intensity decreased and the assembly state lagged behind in Cep57-depleted cells (Figure 6A and 6B). Overexpression of centrosome-localized Cep57-N-terminus (Cep57(1-388)-GFP), which lacks a microtubulebinding domain and inhibits the centrosome localization of endogenous Cep57, induced a similar microtubule assembly defect (Figure 6C and 6D). These data sug-

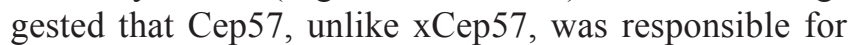
centrosome MTOC activity and microtubule assembly. In addition, the mean fluorescence intensity of half-spindle microtubules in Cep57-depleted cells decreased by $30 \%$ compared with control cells (Figure 6E). Cep57 depletion also resulted in a shortening of spindle length $(20 \%$; Figure 6F), which is consistent with a previous study [16].

A direct interaction of exogenous Cep57 and microtubules has been shown previously [16], but there was no evidence for microtubule localization of endogenous Cep57. The microtubule cooling-rewarming assay showed that endogenous Cep57 localized to the spindle poles and microtubules along with microtubule regrowth (Figure 7A). A small amount of Cep57 was shown to localize along the spindle microtubules by electron microscopy (Supplementary information, Figure S4C). We found that a low level of exogenous Cep57 (only centrosome localized) was sufficient to stabilize micro-
A

$\mathrm{C}^{\frac{5}{2}}$
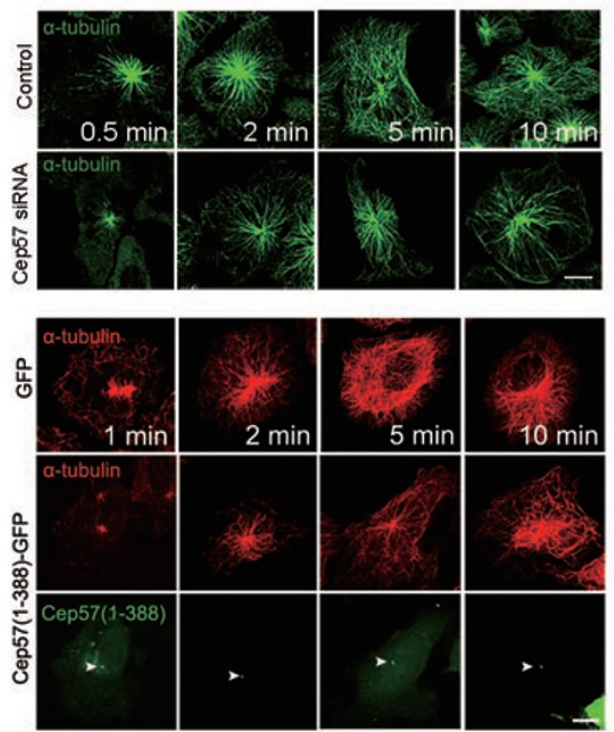

E

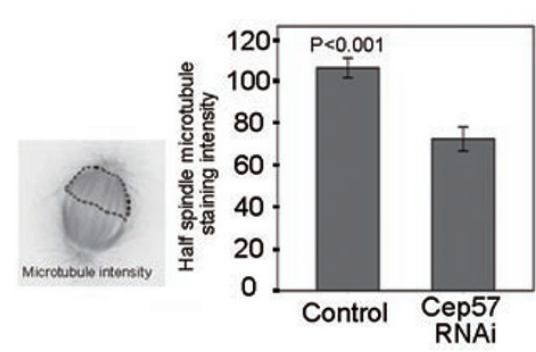

B
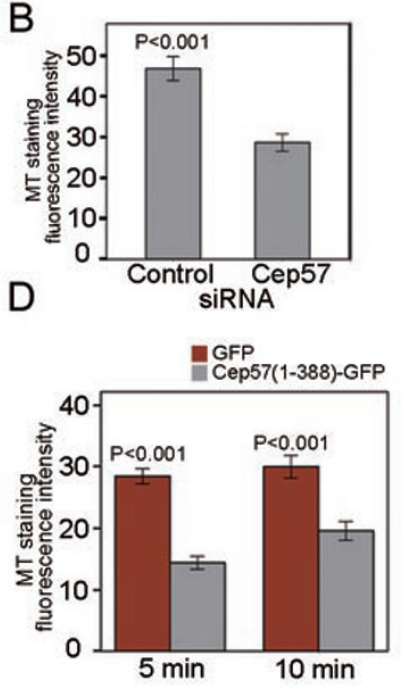

$\mathrm{F}$

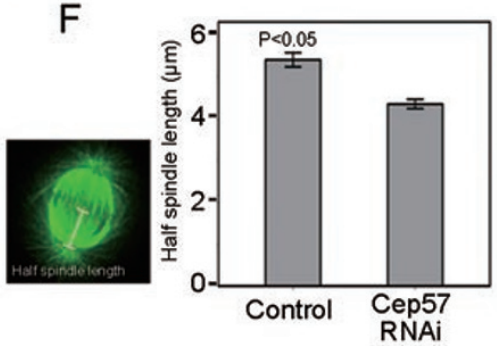

Figure 6 Cep57 functions in spindle microtubule assembly. (A, B) CHO cells were transfected with pSUPER-RFP or pSUPER-RFP-Cep57 and treated with nocodazole. After nocodazole washout, the cells were immunostained for $\alpha$-tubulin (green; A). The graph shows the results of the microtubule regrowth assay at $5 \mathrm{~min}$. Quantification of tubulin immunofluorescence intensities in cells ( $n=3$; $>100$ cells per experiment). Error bars represent mean \pm SEM. (C, D) GFP (upper panels) and Cep57 (1-388)-GFP (middle and lower panels) expressing cells were subjected to a microtubule recovery assay. Arrowheads indicate the centrosome localization of Cep57 (1-388)-GFP. Bar, $10 \mu \mathrm{m}$. The graph shows the results of the microtubule regrowth assay at 5 and $10 \min (n=3 ;>100$ cells per experiment). Error bars represent mean \pm SEM. (E) Quantification of spindle microtubule intensity indicated by $\alpha$-tubulin staining in siRNA-treated cells ( $n=3 ;>75$ cells per experiment). Error bars represent mean \pm SEM. (F) Half spindle lengths indicated by $\alpha$-tubulin staining were measured in control and Cep57-depleted cells ( $n=3 ;>60$ cells per experiment). Error bars represent mean \pm SEM. 

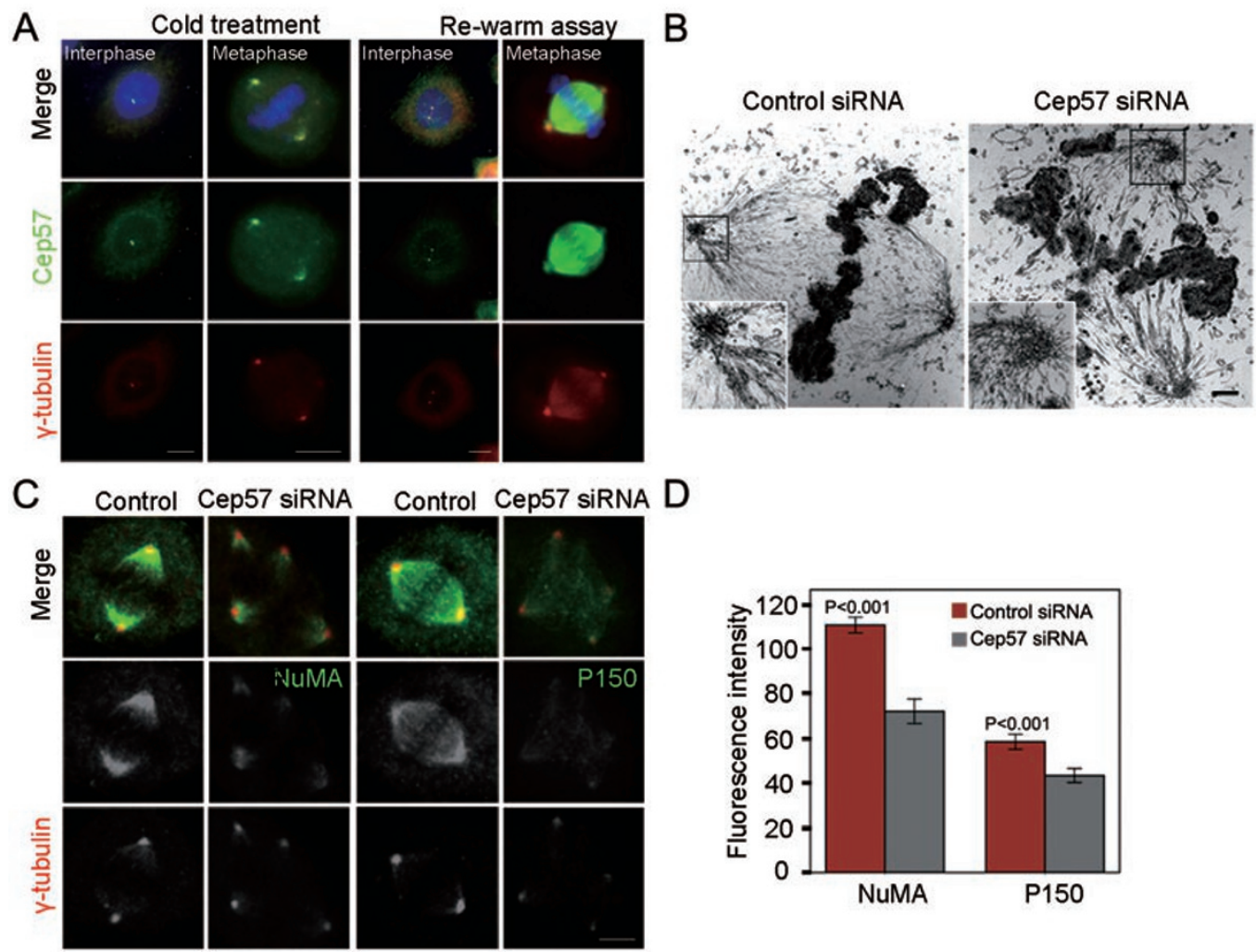

Figure 7 Cep57 binds spindle microtubules and contributes to spindle microtubule organization. (A) HeLa cells in interphase and mitosis were incubated on ice for $1 \mathrm{~h}$ and then warmed to $37^{\circ} \mathrm{C}$ for $10 \mathrm{~min}$. The recovery state was studied by immunofluorescence using anti-Cep57 (green) and $\gamma$-tubulin (red) antibodies. (B) Electron microscopy images of the representative control and Cep57 RNAi spindle. Insets show high magnification images of spindle poles. Bars, $1 \mu \mathrm{m}$. (C, D) HeLa cells treated with siRNAs were stained for NuMA (green), P150 (green) and $\gamma$-tubulin (red). Bars, $5 \mu$ m. Fluorescence intensities in bipolar spindles were measured within a half spindle ( $\mathbf{D}, n=3 ;>100$ cells per experiment). Error bars represent mean \pm SEM.

tubules (Supplementary information, Figure S4D and S4E). Furthermore, compared with normal cells, spindle microtubules were at a lower density and disrupted in Cep57-depleted cells under electron microscope (Figure $7 \mathrm{~B}$ and Supplementary information, Figure S4F). Thus, the endogenous Cep57 on the spindle microtubules might also serve as a stabilization factor of microtubule organization.

Immunoprecipitation experiments showed that Cep57 interacted with a dynein subunit, p150 (Figure 1D), which is responsible for the spindle microtubule minus end localization of NuMA [9]. Therefore, we investigated the localization of these spindle pole-focusing proteins in Cep57-depleted cells. In Cep57 siRNA-treated cells, they were still localized to all the spindle poles, but the intensities of NuMA and p150 labeling on the spindle poles were weakened (Figure 7C and 7D), which is consistent with the previous study of $\mathrm{xCep57}$ [17], while another spindle pole focusing protein TPX2 was not significantly affected (Supplementary information, Figure S5). The loss of NuMA weakened the focusing forces within the minus ends of microtubules [9], which in turn exacerbated the process of spindle pole fragmentation.

\section{Discussion}

We have shown that Cep57 is a PCM component and its interaction with NEDD1 is essential for its centrosome localization. Previous studies have shown that Cep57, as a centrosome protein, has an unconventional $\mathrm{N}$-terminal centrosome-localization domain and can form dimers through this domain [16]. However, it was still unknown how Cep57 localized to the centrosome. Our biochemical studies found that Cep57 interacted with the $\gamma$-TuRC component NEDD1 through its NTD (Figure 2C and 2D). It has previously been suggested 
that NEDD1 interacts with the $\gamma$-TuRC through its CTD, whereas the NTD corresponded to its centrosome attachment [19]. The NEDD1-NTD contains repeated WD40 motifs, which usually act as a protein-protein interaction site [27]. An overexpressed NEDD1-NTD mutant had a diffused cytoplasm localization pattern [21] and blocked the centrosome localization of endogenous Cep57 in a dominant-negative manner (Figure 3K). Combined with the NEDD1-FL overexpression and RNAi experiments, our data suggest the critical role of NEDD1 in the recruitment of Cep57 to the centrosome.

In addition to centrosome localization, exogenous Cep57 localized to microtubules and resulted in microtubule bundling. By immunoelectron mircroscopy, we observed that endogenous Cep57 was localized along the spindle microtubules (Supplementary information, Figure S4C). The molecular mechanism of Cep57 as a MAP is still vague. The $\mathrm{C}$-terminal of Cep57 was reported to contain a novel microtubule-binding domain with no sequence similarity with classical domains [16]. Bioinformatical analysis showed that the ratio of positively charged to negatively charged residues in the C-terminus of Cep57 is nearly 2:1. The isoelectric point (pI) value is as high as 9.62. This kind of characteristic amino-acid composition was also found in other MAPs, such as Tau, MAP1A and MAP2 [28, 29] and has been hypothesized to enable MAPs to electrostatically bind to the negatively charged surface of microtubules. In view of the fact that Cep57 could form homodimers through its N-terminus [16], we speculate that it probably acts as a cross-linker between microtubules. This may explain the microtubule-bundling phenotype induced by Cep57 overexpression. Our results showed that exogenous Cep57, with a low expression level (only the centrosome-localized pattern could be detected by immunofluorescence), helped microtubules to resist nocodazole treatment (Supplementary information, Figure S4D and S4E), which implies a role for the endogenous spindle microtubuleassociated Cep57 in spindle microtubule stabilization. Microtubule recovery experiments showed that depletion of Cep57 decreased the assembly rate and density of the centrosome microtubules, which suggests that Cep57, as a $\gamma$-TuRC-binding protein, may also be involved in the initial process of centrosome microtubule assembly.

In the present study, we have revealed the novel functions of Cep57 in maintaining spindle integrity. Knockdown of Cep57 leads to mitosis delay and multipolar spindles, which is due to PCM fragmentation. Our data suggest that Cep57 governs spindle bipolarity through stabilizing microtubules and centrosomes during mitosis. It is known that optimal spindle organization requires balanced microtubule structure and motor forces [30]. As we mentioned above, Cep57 could form homodimers and cross-link microtubules. Moreover, Cep57 also associates with NEDD1 and is localized to the minus end of the spindle microtubule. Thus, Cep57 might tether the minus ends of spindle microtubules together. In addition, knockdown of Cep57 leads to a decrease in the spindle pole localization of another spindle pole microtubulefocusing protein, NuMA [31]. Thus, combined defects of Cep57 and NuMA might result in force imbalances within spindle microtubule minus ends, which would be expected to have deleterious effects on spindle structure. On the other hand, after the cell enters mitosis, the centrosome expands by recruiting numerous PCM proteins. At the same time, the pulling and pushing forces mediated by microtubules increase to ensure the separation of centrosome asters and bipolar spindle formation [3]. The PCM proteins must assemble properly to form a strong structure that can withstand the spindle microtubule-based tensions. Depletion of several PCM stabilizing proteins, including Kizuna [5], Cep72 [32] and Cep90 [33], induces microtubule-dependent centrosome fragmentation. Cep57, as a component of saltstripped centrosome scaffolds [15], interacts with other PCM proteins like p150 (Figure 1D), RanBMP [13] and Cep152 [34], which suggests that Cep57 may also serve as a PCM stabilization component at the spindle pole to protect the expanded centrosome from collapse. Depletion of Cep57 could therefore cause spindle microtubule disorganization and PCM instability, which would cause some PCMs to be pulled out by microtubule-mediated forces. Those PCM fragments do not contain centrioles but are able to recruit other PCM components and nucleate microtubules [35], eventually forming new spindle poles.

Recently, cep 57 loss-of-function mutations were identified as a cause of MVA syndrome, which is characterized by mosaic aneuploidies and predisposition to cancer. Individuals with MVA have random gains and losses of chromosomes in their cells [18]. Our results may provide a clue to the etiology of MVA. However, it should be noticed that Cep57 may have other currently unknown functions that could also contribute to the phenotypes observed in MVA individuals.

In summary, the loss of Cep57 disrupts the balance of forces within the spindle poles and has an adverse effect on spindle microtubule organization, which in turn results in disorganized spindle morphology (Figure 8). Thus, Cep57 is important for the maintenance of correct chromosome numbers during the cell cycle, which may provide an explanation of the cause of MVA syndrome. 

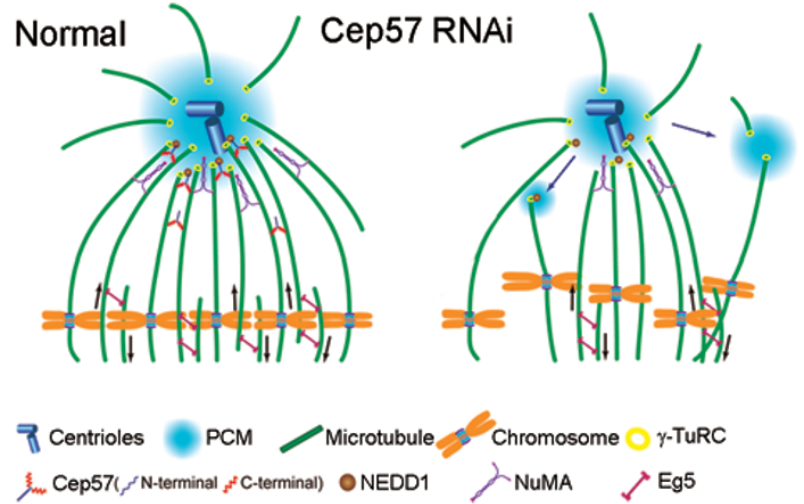

Figure 8 Schematic model highlighting the role of Cep57 in robust spindle architecture establishment. Cep57 acts as a microtubule and PCM stabilization factor at the centrosome and along the spindle microtubules. The loss of Cep57 disrupts the balance of forces within the spindle poles and spindle microtubule organization, which in turn results in disorganized spindle morphology, including multipolar spindles and unaligned chromosomes.

\section{Materials and Methods}

\section{Cell culture, transfections and cell synchronization}

All cell lines used were grown in DMEM (Gibco) with $10 \%$ fetal bovine serum (Gibco) at $37{ }^{\circ} \mathrm{C}$ in an atmosphere of $5 \% \mathrm{CO}_{2}$. Transient transfection was carried out with jetPEI (Polyplus transfection) or Lipofectamine ${ }^{\mathrm{TM}} 2000$ (Invitrogen) according to the manufacturer's instructions.

For mitosis synchronization, HeLa cells were treated with 2.5 $\mathrm{mM}$ thymidine (Sigma) for $24 \mathrm{~h}$ and then released for 11-12 $\mathrm{h}$. For MA treatment, cells were synchronized at the G1/S boundary with thymidine, and then released for $7 \mathrm{~h}$, followed by treatment with $100 \mu \mathrm{M}$ MA (Sigma) for $4 \mathrm{~h}$. For HU (Sigma) treatment, CHO cells were treated with $2 \mathrm{mM} \mathrm{HU}$ for $72 \mathrm{~h}$. For MG132 treatment, MG132 $(20 \mu \mathrm{M})$ was added into cell culture media at $65 \mathrm{~h}$ after NEDD1 siRNA transfection and incubated for $6 \mathrm{~h}$ at $37^{\circ} \mathrm{C}$.

\section{Antibodies}

The full-length mouse Cep57 cDNA from a mouse liver cDNA library was cloned into pET28a (Novagen). The recombinant protein was purified by affinity chromatography on Ni-NTA beads (Invitrogen) under denaturing conditions. Purified protein was injected into mice and rabbits to produce polyclonal antibodies. The rabbit anti-Cep57 antibody was affinity purified against the recombinant GST-Cep5 $7_{332-500}$ fusion protein.

The following antibodies were also used: anti-centrin2 (Santa Cruz Biotechnology), anti-pericentrin (Abcam), anti-GCP2 (Santa Cruz Biotechnology), anti-ninein (Abcam), anti- $\alpha$-tubulin (DM1A, Sigma), anti- $\gamma$-tubulin (GTU88, Sigma), anti- $\gamma$-tubulin (rabbit antiserum, Sigma), anti-p150 (BD Transduction Laboratories), anti-GAPDH (Biolinks), anti-TPX2, anti-NEDD1 (gift from Dr. C Zhang), anti-NuMA (Santa Cruz Biotechology), anti-myc (Millipore) and anti-GFP (rabbit polyclonal antibody, produced by our lab; mouse monoclonal antibody, MBL). The following florescent secondary antibodies were used: Alexa Fluor 488-conjugated goat anti-mouse IgG, Alexa Fluro 488-conjuagated goat anti-rabbit IgG, Alexa Fluro 568-conjugated goat anti-mouse IgG and Alexa Fluro 568-conjugated anti-rabbit IgG (Molecular Probes).

\section{$R N A i$}

Cep57 siRNAs were obtained from GenePharma with the following sequences: 5'-AAGCATGCAGAAATGGAGAGG-3', 5'-AACCATCAAGGTCTAATGGAA-3' and 5'-AACCAAATAACTAAAGTTCGA-3'. hNuf2 siRNA: 5'-AAGCATGCCGTGAAACGTATA-3' [26]. Negative control siRNA: 5'-UUCUCCGAACGUGUCACGUTT-3'. The pSUPER-RFP encoding Cep57 and control siRNAs were constructed according to the pSUPER RNAi System protocols. The pSUPER-NEDD1 plasmid was kindly provided by Dr C Zhang [20].

\section{IP and immunoblotting}

$24 \mathrm{~h}$ after transfection, cells were washed three times with PBS and lysed in lysis buffer (50 mM HEPES pH 7.4, 1 mM EDTA, $150 \mathrm{mM} \mathrm{NaCl}, 0.5 \%$ Triton X-100, $2 \mathrm{mM}$ PMSF, $10 \mu \mathrm{g} / \mathrm{ml}$ Aprotinin and $5 \mu \mathrm{g} / \mathrm{ml}$ Pepstatin A) on ice for $30 \mathrm{~min}$. The lysates were centrifuged at $20000 \times g$ for $10 \mathrm{~min}$ and the supernatant was then mixed with the appropriate antibody. The mixtures were incubated overnight at $4{ }^{\circ} \mathrm{C}$, followed by $2 \mathrm{~h}$ incubation with protein A-Sepharose beads (Pharmacia). After five washes with the lysis buffer, the bound immunocomplexes were eluted by boiling in SDS-PAGE loading buffer and subjected to immunoblotting analysis as described previously [36]. Briefly, the immunoprecipitated complexes were resolved by SDS-PAGE and transferred to PVDF membrane (Millipore). The membranes were incubated with primary antibodies followed by HRP-conjugated or AP-conjugated secondary antibodies.

\section{Microscopy}

Cells grown on coverslips were fixed in methanol at $-20{ }^{\circ} \mathrm{C}$ for $7 \mathrm{~min}$ or in $4 \%$ paraformaldehyde with $0.1 \%$ Triton X-100 at room temperature for $15 \mathrm{~min}$. Alexa 488- and Alexa 568-conjugated highly cross absorbed goat anti-mouse and goat anti-rabbit $\operatorname{IgG}$ were used as secondary antibodies. The fixed cell samples were observed at room temperature under a TCS SP2 laser-scanning confocal microscope, with a $100 \times 1.4$ NA Apo oil immersion lens on an upright microscope (DM IRBE; Leica), or under a IX 71 inverted fluorescent microscope (Olympus), equipped with a $60 \times$ 1.4 NA Apo oil immersion lens (Leica). Images were acquired by Leica Confocal Software and DP controller software (Olympus). Image processing was performed in DP manager (Olympus) and Photoshop (CS2; Adobe).

For FRET assays, the $488 \mathrm{~nm}$ laser of a LSM 710 (Zeiss) was used to excite Alexa Fluor 488, and the $568 \mathrm{~nm}$ laser was used to excite and bleach Alexa Fluor 568. The centrosome region and randomly chosen cytoplasmic regions were drawn for bleaching. The FRET images and efficiencies were generated and calculated by Zeiss LSM 710 software.

For time-lapse microscopy, HeLa cells transfected with Cep57 siRNA and $\alpha$-tubulin-pCAsalGFP [37] were randomly selected and placed in a $37{ }^{\circ} \mathrm{C}$ heated microscope chamber with $5 \% \mathrm{CO}_{2}$. Live-cell video microscopy was carried out on a PerkinElmer UltraVIEW VoX system, with an inverted microscope (ECLIPSE TiE, Nikon) equipped with a CFI Plan Apochromat VC 60XH N.A. 
1.40, W.D.0.13 mm lens (Nikon). The time-lapse confocal images were acquired with Volocity Imaging Software (PerkinElmer).

The immunoelectron microscopy was performed according to a pre-embedding immunogold staining protocol. HeLa cells were grown on coverslips, extracted for $5 \mathrm{~min}$ in extraction buffer (PEM buffer, $10 \mu \mathrm{m}$ taxol, $0.1 \%$ Triton $\mathrm{X}-100$ ) and fixed with $2 \%$ PFA and $0.1 \%$ GA in PBS. Cells were incubated with mouse antiCep57 antibody and Nanogold-conjugated anti-mouse IgG antibody (Sigma) overnight at $4{ }^{\circ} \mathrm{C}$. For spindle ultrastructure observation, the HeLa cells were transfected with pSUPER-RFP (control) or pSUPER-RFP-Cep57. The cells were processed according to a standard EM sample preparation procedure [38], and dehydrated and embedded in Epon resin. The samples were observed with a transmission electron microscope (JEOL, JEM 1010).

\section{Measurement and statistical analysis}

Spindle lengths were measured by Image J (NIH) software. The fluorescence intensity was measured by Scion image (NIH) software. Statistical methods employed by SPSS software are described in the figure legends. Statistical significance was determined by the Student's $t$-test.

\section{Acknowledgments}

We thank Chuanmao Zhang, Xiaoyan Zhang and Jingyan Fu for TPX-2 antibody and NEDD1 plasmids; Masatoshi Takeichi and Wenxiang Meng for the $\alpha$-tubulin-pCAsalGFP plasmid. This work was supported by the National Natural Science Foundation of China (30971433, 31171283) and the National Basic Research Program of China (973 Program; 2010CB833705).

\section{References}

1 Blagden SP, Glover DM. Polar expeditions - provisioning the centrosome for mitosis. Nat Cell Biol 2003; 5:505-511.

2 Zheng Y, Wong ML, Alberts B, Mitchison T. Nucleation of microtubule assembly by a gamma-tubulin-containing ring complex. Nature 1995; 378:578-583.

3 Palazzo RE, Vogel JM, Schnackenberg BJ, Hull DR, Wu X. Centrosome maturation. Curr Top Dev Biol 2000; 49:449470 .

4 Thein KH, Kleylein-Sohn J, Nigg EA, Gruneberg U. Astrin is required for the maintenance of sister chromatid cohesion and centrosome integrity. J Cell Biol 2007; 178:345-354.

5 Oshimori N, Ohsugi M, Yamamoto T. The Plk1 target Kizuna stabilizes mitotic centrosomes to ensure spindle bipolarity. Nat Cell Biol 2006; 8:1095-1101.

6 Yang S, Liu X, Yin Y, Fukuda MN, Zhou J. Tastin is required for bipolar spindle assembly and centrosome integrity during mitosis. FASEB J 2008; 22:1960-1972.

7 Heald R. Motor function in the mitotic spindle. Cell 2000; 102:399-402.

8 Andersen SS. Spindle assembly and the art of regulating microtubule dynamics by MAPs and Stathmin/Op18. Trends Cell Biol 2000; 10:261-267.

9 Merdes A, Heald R, Samejima K, Earnshaw WC, Cleveland DW. Formation of spindle poles by dynein/dynactin-dependent transport of NuMA. J Cell Biol 2000; 149:851-862.

10 Garrett S, Auer K, Compton DA, Kapoor TM. hTPX2 is re- quired for normal spindle morphology and centrosome integrity during vertebrate cell division. Curr Biol 2002; 12:20552059.

11 Kwok BH, Yang JG, Kapoor TM. The rate of bipolar spindle assembly depends on the microtubule-gliding velocity of the mitotic kinesin Eg5. Curr Biol 2004; 14:1783-1788.

12 Wordeman L, Wagenbach M, von Dassow G. MCAK facilitates chromosome movement by promoting kinetochore microtubule turnover. J Cell Biol 2007; 179:869-879.

13 Meunier S, Navarro MG, Bossard C, et al. Pivotal role of translokin/CEP57 in the unconventional secretion versus nuclear translocation of FGF2. Traffic 2009; 10:1765-1772.

14 Bossard C, Laurell H, Van den Berghe L, Meunier S, Zanibellato $\mathrm{C}$, Prats $\mathrm{H}$. Translokin is an intracellular mediator of FGF-2 trafficking. Nat Cell Biol 2003; 5:433-439.

15 Andersen JS, Wilkinson CJ, Mayor T, Mortensen P, Nigg EA, Mann M. Proteomic characterization of the human centrosome by protein correlation profiling. Nature 2003; 426:570-574.

16 Momotani K, Khromov AS, Miyake T, Stukenberg PT, Somlyo AV. Cep57, a multidomain protein with unique microtubule and centrosomal localization domains. Biochem J 2008; 412:265-273.

17 Emanuele MJ, Stukenberg PT. Xenopus Cep57 is a novel kinetochore component involved in microtubule attachment. Cell 2007; 130:893-905.

18 Snape K, Hanks S, Ruark E, et al. Mutations in CEP57 cause mosaic variegated aneuploidy syndrome. Nat Genet 2011; 43:527-529.

19 Haren L, Remy MH, Bazin I, Callebaut I, Wright M, Merdes A. NEDD1-dependent recruitment of the gamma-tubulin ring complex to the centrosome is necessary for centriole duplication and spindle assembly. J Cell Biol 2006; 172:505-515.

20 Zhang X, Chen Q, Feng J, et al. Sequential phosphorylation of Nedd1 by Cdk1 and Plk1 is required for targeting of the gammaTuRC to the centrosome. J Cell Sci 2009; 122:22402251.

21 Manning JA, Shalini S, Risk JM, Day CL, Kumar S. A direct interaction with NEDD1 regulates gamma-tubulin recruitment to the centrosome. PLoS One 2010; 5:e9618.

22 Ganem NJ, Godinho SA, Pellman D. A mechanism linking extra centrosomes to chromosomal instability. Nature 2009; 460:278-282.

23 Kuriyama R, Terada Y, Lee KS, Wang CL. Centrosome replication in hydroxyurea-arrested $\mathrm{CHO}$ cells expressing GFPtagged centrin2. J Cell Sci 2007; 120:2444-2453.

24 Mayer TU, Kapoor TM, Haggarty SJ, King RW, Schreiber SL, Mitchison TJ. Small molecule inhibitor of mitotic spindle bipolarity identified in a phenotype-based screen. Science 1999; 286:971-974.

25 Kapoor TM, Mayer TU, Coughlin ML, Mitchison TJ. Probing spindle assembly mechanisms with monastrol, a small molecule inhibitor of the mitotic kinesin, Eg5. J Cell Biol 2000; 150:975-988.

26 DeLuca JG, Moree B, Hickey JM, Kilmartin JV, Salmon ED. hNuf2 inhibition blocks stable kinetochore-microtubule attachment and induces mitotic cell death in HeLa cells. $J$ Cell Biol 2002; 159:549-555.

27 Li D, Roberts R. WD-repeat proteins: structure characteristics, biological function, and their involvement in human dis- 
eases. Cell Mol Life Sci 2001; 58:2085-2097.

28 Al-Bassam J, Ozer RS, Safer D, Halpain S, Milligan RA. MAP2 and tau bind longitudinally along the outer ridges of microtubule protofilaments. J Cell Biol 2002; 157:1187-1196.

29 Cravchik A, Reddy D, Matus A. Identification of a novel microtubule-binding domain in microtubule-associated protein 1A (MAP1A). J Cell Sci 1994; 107 (Pt 3):661-672.

30 Kline-Smith SL, Walczak CE. Mitotic spindle assembly and chromosome segregation: refocusing on microtubule dynamics. Mol Cell 2004; 15:317-327.

31 Silk AD, Holland AJ, Cleveland DW. Requirements for NuMA in maintenance and establishment of mammalian spindle poles. J Cell Biol 2009; 184:677-690.

32 Oshimori N, Li X, Ohsugi M, Yamamoto T. Cep72 regulates the localization of key centrosomal proteins and proper bipolar spindle formation. EMBO J 2009; 28:2066-2076.

33 Kim K, Rhee K. The pericentriolar satellite protein CEP90 is crucial for integrity of the mitotic spindle pole. J Cell Sci 2011; 124:338-347.

34 Hutchins JR, Toyoda Y, Hegemann B, et al. Systematic analysis of human protein complexes identifies chromosome segregation proteins. Science 2010; 328:593-599.

35 Rusan NM, Rogers GC. Centrosome function: sometimes less is more. Traffic 2009; 10:472-481.

36 Li H, Guo Y, Teng J, Ding M, Yu AC, Chen J. 14-3-3gamma affects dynamics and integrity of glial filaments by binding to phosphorylated GFAP. J Cell Sci 2006; 119:4452-4461.

37 Meng W, Mushika Y, Ichii T, Takeichi M. Anchorage of microtubule minus ends to adherens junctions regulates epithelial cell-cell contacts. Cell 2008; 135:948-959.

38 Teng J, Takei Y, Harada A, Nakata T, Chen J, Hirokawa N. Synergistic effects of MAP2 and MAP1B knockout in neuronal migration, dendritic outgrowth, and microtubule organization. J Cell Biol 2001; 155:65-76.

(Supplementary information is linked to the online version of the paper on the Cell Research website.) 